\title{
Influence of Bulk Convection on Freckle Formation
}

\section{in Castings}

\author{
V. Sahai, R. A. Overfelt, and P. Banerjee \\ Space Power Institute, 231 Leach Center, Auburn University, Auburn, Alabama 36849
}

\begin{abstract}
In recent years, there has been an increased usage of single crystal superalloys in high temperature components because of the requirements for high creep resistance. For these complex alloys, it is necessary to understand the molten alloy convective phenomena that can cause deleterious grain defects. In particular, it is believed that the penetration of the bulk convection into the mushy zone can assist in the initiation of upward flowing liquid jets, i.e. channels, which sweep dendritic fragments out of the mushy zone and into the bulk liquid. These fragments can then serve as nuclei for growth of spurious grains that lead to defects called freckles. In the present work, a detailed quasi-steady state, axisymmetric FIDAP model is utilized to simulate the thermal buoyancy convection that occurs during directional solidification. The governing equations for the alloy and the crucible are solved subject to appropriate boundary conditions to determine the velocity and temperature distributions. The results obtained show how the major process parameters such as solidification velocity, and crucible diameter influence convection in the mushy zone. Finally, the numerical results are shown to agree with existing experimental data on freckle formation in metal alloy systems.
\end{abstract}

\section{INTRODUCTION}

New alloys and turbine blade designs for next generation aircraft promise to continue pushing the limits of manufacturing technology by requiring ever more complex components to be produced. Unfortunately, the more complex the geometry of the turbine blade, the lower the production yield and the higher the cost of the casting. In fact, scrap rates of $40 \%$ are common with some single crystal designs due to grain defects referred to as slivers, equiaxed grains, freckles, and freckle chains. In addition to the costs of recurring scrap, these spurious grain defects can significantly delay the introduction of new casting designs as foundry personnel are forced to empirically adjust their processes prior to full-scale production. Currently, the fundamental solidification phenomena controlling the origins of these defects are only qualitatively understood.

In general, single crystal turbine blades are cast in a specialized vacuum furnace with two compartments, a hot zone and a cold zone as shown in Figure 1(a) below. A baffle is used to separate these chambers and minimize energy exchange between them except for a circular opening for the mold. To increase productivity, multiple parts are usually cast in a cluster of molds. Initially, the mold cluster sits inside the hot zone above the melting temperature. Liquid metal is then poured into the mold, and the mold is gradually withdrawn into the cold

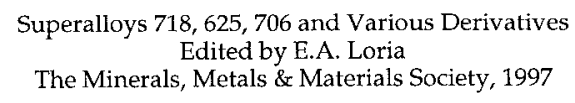


zone to establish directional solidification conditions. Single crystal growth of highly aligned dendrites as shown in Figure 1(b) is achieved through a starter block and a grain selector to ensure that the casting originates from a single grain. A copper chill plate is usually used to improve the vertical heat flux and columnar grain growth in the starter block.

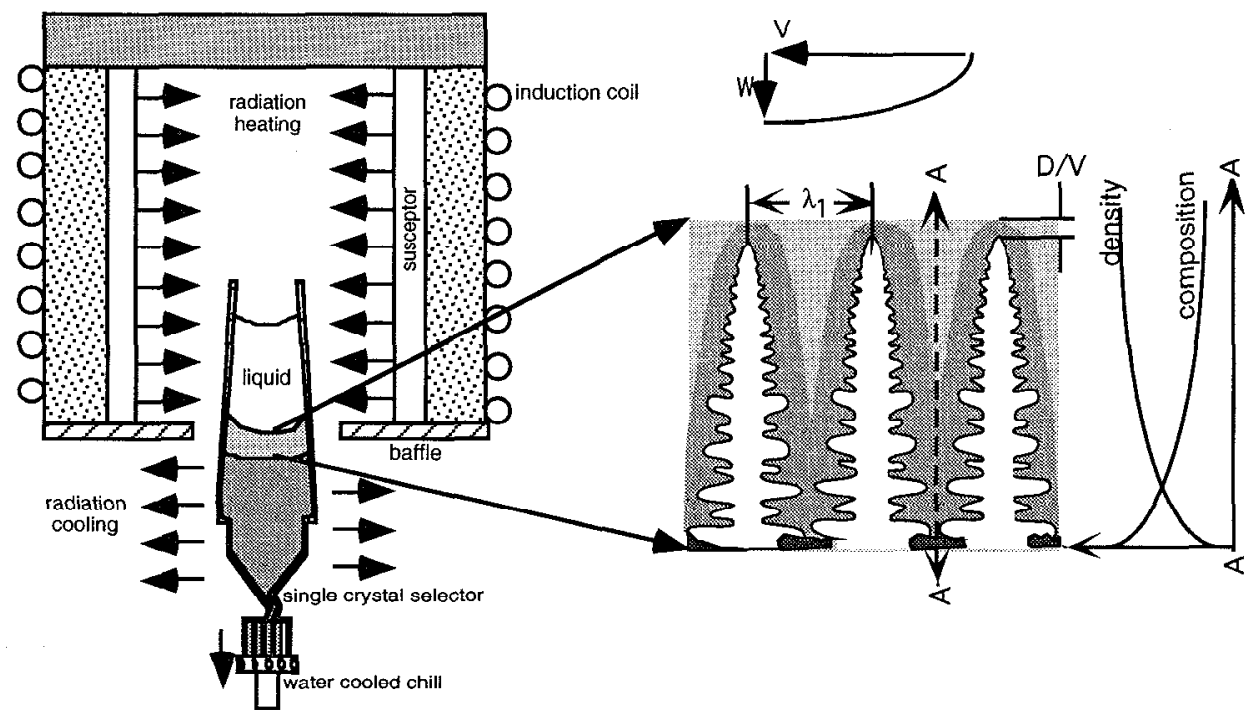

(a)

(b)

Figure 1.(a) Process schematic of single crystal investment casting process

(b) Highly aligned columnar growth with interdendritic liquid of less density than bulk liquid.

Single crystal turbine blades produced by this process often contain defects called freckle chains on their outer surface. Freckle chains are extended trails of macrosegregation containing excess eutectic material, second phase particles, and small equiaxed grains. Giamei and Kear [1] and Copley et al.[2] showed that freckle chains are caused by upwardly flowing jets originating in the mushy zone. Although the exact mechanism for debris generation is unknown [3], these jets are believed to be responsible for causing the erosion of dendrite arms and leaving a trail of dendritic debris. Copley et al.[2] showed that the location of the freckles is influenced by mushy zone orientation and shape.

Sample and Hellawell [4] deduced a phenomenological model which described the initiation of freckle chains. According to this model, the key event involves a perturbation, possibly caused by the convection from the bulk fluid, which initiates a plume. The plume draws the surrounding low density segregated liquid from the mushy zone. This flow causes a local depression in the liquidus and produces an open channel, thus providing a self-sustaining path for fceding the plume. McCay [5] postulated that the pluming could be the result of a Rayleigh-Bernard instability at the interface between the bulk fluid and the mushy zone. These plumes are postulated to be the initiating mechanisms for freckle chains.

The formation of freckles may be enhanced by low thermal gradients, low solidification velocities, and a large density inversion of the segregated liquid. Copley et al.[2] suggested that the casting's cooling rate should be above a critical cooling rate or freckles would be likely. Sarazin and Hellawell[6] calculated mushy zone Rayleigh numbers to characterize the frecklc formation tendency. They showed that increases in interdendritic spacing or density differences caused by temperature and/or composition changes enhance the potential for freckle formation. Pollock et al. [7] also investigated the dominating influence of cooling rate and resultant primary dendrite arm spacing on freckle chain formation in a series of directionally solidified nickel-based superalloys. Their results showed clearly that decreasing the interdendritic spacing would decrease the permeability of the mushy zone and retard feeding and development of plumes essential for freckle formation. 
Muller [8] in his monograph on crystal growth extensively examined the natural convection in simply shaped cavities and determined the critical Rayleigh numbers for the onset of steady convection and for transition from steady to unsteady convection over a wide range of aspect ratios for a top seeded Bridgman apparatus. McCay et al.[9] studied the thermosolutal convection in the presence of a mushy zone in the directional solidification of aqueous ammonium chloride materials by optical and laser particle tracking techniques. Dependent upon the Rayleigh number, they noticed both Bernard type cellular convection and pluming flows. The presence of a mushy zone increased resistance to flow and raised the critical Rayleigh numbers for onset of both the cellular and plume-like flows. These trends are consistent with the results described by Sample and Hellawell[4] and Pollock[7]. This work of McCay et al. [9] is especially useful in understanding the convection regimes and provides insight for developing mathematical and computational models of freckle formation.

Heinrich et al.[10] developed a mathematical model which simulated the convection in directionally solidified binary alloys. Their results showed that the interdendritic convection in the mushy zone is mainly driven by the convection in the bulk liquid. These results were confirmed by a finite element analysis of a simple 2-D rectangular cavity with adiabatic side walls performed by Felicelli et al.[11]. Their predictions of the location of channels agreed with known experimental observations. Motakef[12] developed scaling laws for the convective flow for bottom seeded vertical Bridgman crystal growth and showed that increases in the crucible Rayleigh number due to the increases in the temperature gradient or length scale exponentially increases the strength of the convective flow. Sahai [13] showed that increases in the crucible diameter also enhances the size and strength of the convective cells in a Bridgman apparatus.

In a separate study[14], the authors of the present paper developed a computational model of an investment cluster of a nickel based superalloy PWA 1484 cast at HOWMET Corporation. This study showed that mushy zone orientation and the volumes of the different parts of the casting have a major influence on freckle formation. It also confirmed the importance of cooling rates in the development of freckles.

The above-mentioned study concluded that the interaction between the low-density segregated liquid in the mushy zone and the natural convection in the bulk liquid must be clearly understood if quantitative predictions of freckle chains in complex turbine components are to be realized. The chemistries of alloys like PWA 1484 are very complex and there are little data available on the phase diagram or segregation coefficients for this alloy. The focus will therefore be placed on understanding the thermal behavior of these systems. In this paper, a computational model is presented which examines the thermal buoyancy convection during the directional solidification of nickel-based superalloys. The model will be used to study the penetration of the bulk convection into the mushy zone. The effect of the major process parameters such as aspect ratio (changes in diameter), withdrawal rate (which influences the solidification rate), and thermal gradient were studied. In this work results showing the effects of diameter and withdrawal rate are shown. The predictions from the model will then be compared to known experimental results on freckle formation.

\section{Model Description}

The heat and mass transfer processes that occur during directional solidification are extremely complicated. In order to make the problem tractable, a somewhat simplified numerical model is considered in the present work. This section describes the geometry of the model used and presents the appropriate governing equations. In the directional solidification process, there is a continuous translation of the ampoule through the furnace which results in a time dependent process. Between the initial and final transients, however, Su et al.[15] (for aluminum-copper alloys), Overfelt et al.[16] (for Inconel 718), and Wang [17] (for galliumdoped germanium) found that there exists a region where the crystal solidification rate equals the ampoule translation rate. The dendrite arm spacing remains constant in this regime. The present model describes the convection that occurs in this regime. The translation of the ampoule can be simulated by assuming that the melt of a given composition is introduced at the top of the ampoule at a uniform velocity. At the same time, the solidified crystal of the same 
average composition is continuously removed at the bottom with a growth rate equal to melt input velocity. The ampoule is assumed to be sufficiently long so that the transients in velocity, temperature, pressure, and concentration due to the decrease in melt length and displacement of the ampoule can be safely neglected. The assumption of a long ampoule also justifies the neglect of thermal end effects and avoids the initial and final transients that occur during crystal growth.

One of the primary purposes of this study was to consider different diameter-to-length ratios. In order to avoid numerical instabilities due to application of inlet conditions, it was necessary to add an additional adiabatic zone at the top. This extended length model ensures that the bulk convection cell will not distort the flow at the inlet. The dimensions associated with this model are given in Table 1.

\section{Table 1. Nondimensional Model Parameters}

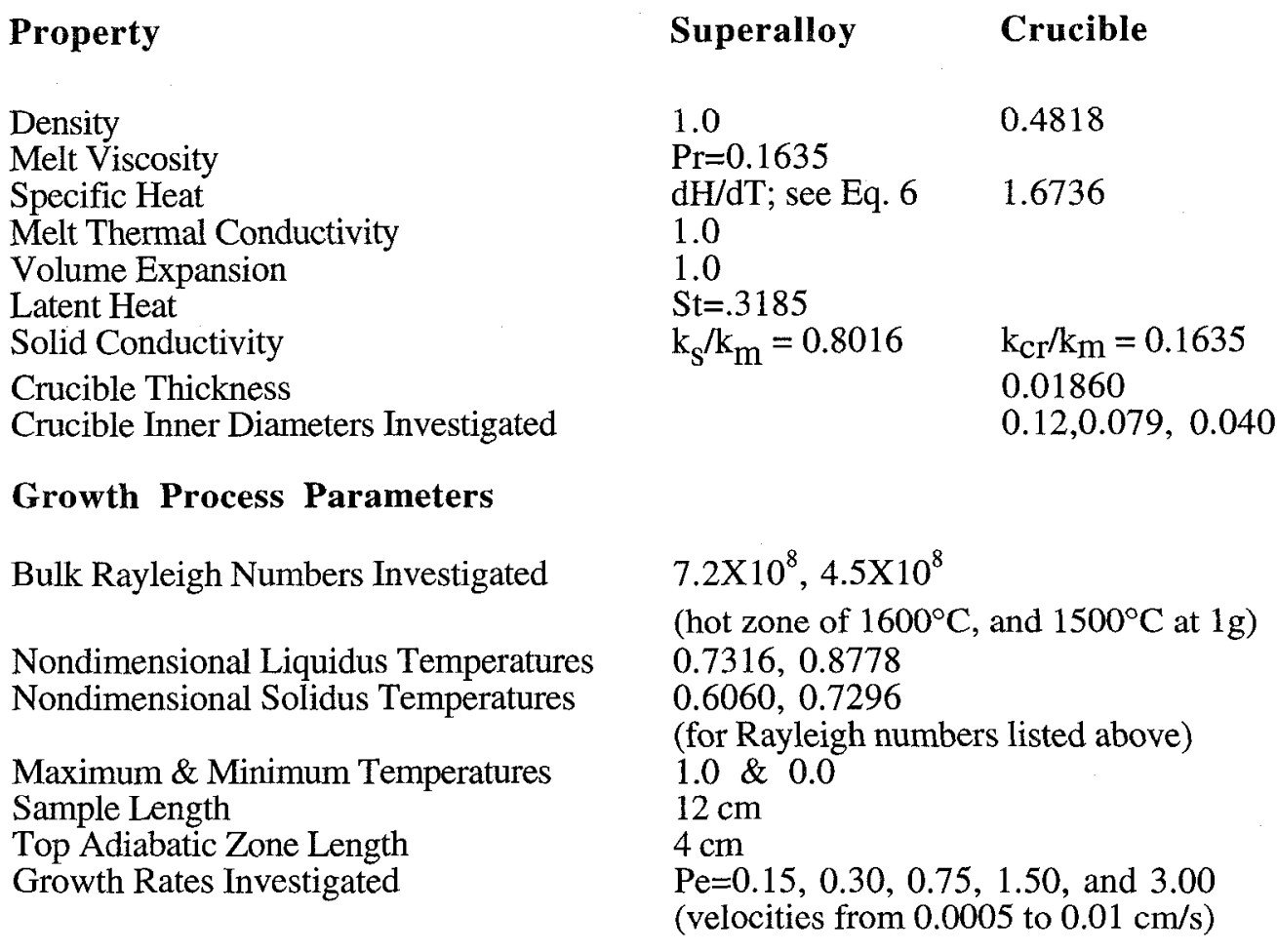

To model both the convective and heat transfer processes which occur, the applicable set of continuity, motion, and energy equations for the growth material and ampoule are simplified using the ideas of the quasi-steady state model described above. The melt material is considered to be incompressible and Newtonian, and the velocity and temperaturc fields are assumed to be axisymmetric. Coupling of the thermosolutal buoyancy effects in the mushy zone with the bulk convection effects is neglected in this work. The solidification of the growth material is treated as a porous medium. The governing equations are thus written in terms of a superficial ('Darcian') velocity which can be defined as: $v=(1-f s(T)) v_{1}$ and $w=(1-f s(T)) w_{1}$ where $\mathrm{fs}(\mathrm{T})$ is the fraction solid and $\mathrm{v}_{1}$ and $\mathrm{w}_{1}$ represent the actual liquid velocities in the radial and axial direction. Since a major theme of the present research is to conduct parametric studies, the equations are written in their nondimensional form by scaling the variables with respect to their characteristic values. The characteristic values used for scaling lengths, 
velocities, and pressures are respectively, $\mathrm{L}, \alpha_{\mathrm{m}} / \mathrm{L}$, and $\left(\rho_{\mathrm{m}} \alpha_{\mathrm{m}}{ }^{2}\right) / \mathrm{L}^{2}$. Here, $\mathrm{L}$ represents the length of the crucible, $\rho_{m}$ is the density of the melt, and $\alpha_{m}$ is the thermal diffusivity of the melt. For the temperature, it is convenient to use the following dimensionless form:

$$
\theta=\frac{\mathrm{T}-\mathrm{T}_{\mathrm{c}}}{\mathrm{T}_{\mathrm{h}}-\mathrm{T}_{\mathrm{c}}}=\frac{\mathrm{T}-\mathrm{T}_{\mathrm{c}}}{\Delta \mathrm{T}}
$$

where $T_{c}$ and $T_{h}$ are respectively the maximum and minimum temperatures in the cold and hot zones of the furnace. To account for the advection of latent heat in the mushy zone, the enthalpy form of the energy equation was used. The enthalpy was nondimensionalized with respect to the product of the melt specific heat and overall temperature difference $\left(\mathrm{c}_{\mathrm{pl}} \Delta \mathrm{T}\right)$. The dimensionless form of the equations governing the mass, momentum, and heat transfer are then

Momentum Equations:

$$
\frac{1}{\mathrm{r}^{*}} \frac{\partial}{\partial \mathrm{r}^{*}}\left(\mathrm{r}^{*} \mathrm{v}^{*}\right)+\frac{\partial}{\partial \mathrm{z}^{*}}\left(\mathrm{w}^{*}\right)=0
$$

$$
\begin{gathered}
1 \\
(1-\mathrm{fs}(\mathrm{T}))^{2}\left(\mathrm{v}^{*} \frac{\left.\partial \mathrm{w}^{*}+\mathrm{w}^{*} \partial \mathrm{w}^{*}\right)}{\partial \mathrm{r}^{*}}\right)=-\frac{\partial \mathrm{p}^{*}}{\partial \mathrm{z}^{*}-\operatorname{Ra} \operatorname{Pr}(\theta-1)} \\
+\frac{\mathrm{Pr}}{(1-\mathrm{fs}(\mathrm{T}))}\left(\frac{1}{\mathrm{r}^{*}} \frac{\partial}{\partial \mathrm{r}^{*}}\left(\mathrm{r}^{*} \frac{\partial \mathrm{w}^{*}}{\partial \mathrm{r}^{*}}\right)+\frac{\partial^{2} \mathrm{w}^{*}}{\partial \mathrm{z}^{*}}\right)-\frac{\operatorname{Pr}}{\mathrm{Da} \mathrm{z}} \mathrm{w}^{*} \\
\frac{1}{(1-\mathrm{fs}(\mathrm{T}))^{2}}\left(\mathrm{v}^{*} \frac{\partial \mathrm{v}^{*}}{\partial \mathrm{r}^{*}}+\mathrm{w} \frac{\partial \mathrm{v}^{*}}{\partial \mathrm{z}^{*}}\right)= \\
-\frac{\partial \mathrm{p}^{*}}{\partial \mathrm{r}^{*}}+\frac{\mathrm{Pr}}{(1-\mathrm{fs}(\mathrm{T}))}\left(\frac{\partial}{\partial \mathrm{r}^{*}}\left(\frac{1}{\mathrm{r}^{*}} \frac{\partial\left(\mathrm{r}^{*} \mathrm{v}^{*}\right)}{\partial \mathrm{r}^{*}}\right)+\frac{\partial^{2} \mathrm{v}^{*}}{\partial \mathrm{z}^{* 2}}\right)-\frac{\operatorname{Pr}}{\mathrm{Da}_{\mathrm{r}}} \mathrm{v}^{*}
\end{gathered}
$$

Energy Equation:

$$
\mathrm{v}^{*} \frac{\partial \mathrm{H}^{*}}{\partial \mathrm{r}^{*}}+\mathrm{w}^{*} \frac{\partial \mathrm{H}^{*}}{\partial \mathrm{z}^{*}}=\frac{1}{\mathrm{r}^{*}} \frac{\partial}{\partial \mathrm{r}^{*}}\left(\mathrm{r}^{*} \frac{\partial\left(\mathrm{k}^{*} \mathrm{H}^{*}\right)}{\partial \mathrm{r}^{*}}\right)+\frac{\partial^{2}\left(\mathrm{k}^{*} \mathrm{H}^{*}\right)}{\partial \mathrm{z}^{* 2}}
$$

Here, $\operatorname{Pr}$ is the Prandtl number $\left(v / \alpha_{\mathrm{m}}\right), \mathrm{Ra}$ is the Rayleigh number $\left(\mathrm{g} \beta_{\mathrm{T}} \Delta \mathrm{T}\right.$ $\left.\mathrm{L}^{3 /} \alpha_{\mathrm{m}} v\right)$, and Da is the Darcy number $\left(\mathrm{K}_{\mathrm{Z}} / \mathrm{L}^{2}, \mathrm{~K}_{\mathrm{r}} / \mathrm{L}^{2}\right)$. The symbols undefined so far include the kinematic viscosity: $v$, the volumetric coefficient of thermal expansion: $\beta_{\mathrm{T}}$, the nondimensional thermal conductivity: $\mathrm{k}^{*}=\left(\mathrm{k} / \mathrm{k}_{\mathrm{II}}\right)$, and the permeability: $\mathrm{K}_{\mathrm{Z}}$ (axial) and $\mathrm{K}_{\mathrm{r}}$ (radial).

To model the effects of phase change from melt to solid, temperature dependent functions were used for the thermal conductivity, permeability, and enthalpy. The thermophysical properties utilized were typical for nickel-based superalloys [18]. The specific heat is computed from the slope of the enthalpy-temperature curve based on the temperature at the point. The enthalpy-temperature curve is constructed such that it reflects the specific heat of 
the solid $\left(c_{\mathrm{ps}}\right)$, liquid $\left(\mathrm{c}_{\mathrm{pl}}\right)$, and the latent heat release $(\Delta \mathrm{H})$ in the mushy zone. The latent heat release in the mushy zone is controlled by the fraction solid curve $f_{S}(T)$ of the growth material. The fraction solid values were determined by using a cooling curve analysis $[14,19]$. The fraction solid curve used in this analysis is shown in Figure 2 . The equation for the enthalpy function can be written as:

$$
H(T)=\int c_{p s} d T+\Delta H\left(1-f_{S}(T)\right)+\int c_{p l} d T
$$

This curve has been nondimensionalized with respect to the product of the melt specific heat and the overall temperature difference. The latent heat is thus nondimensionalized by the Stefan number $\left(c_{\mathrm{pl}} \Delta \mathrm{T} / \Delta \mathrm{H}\right)$.

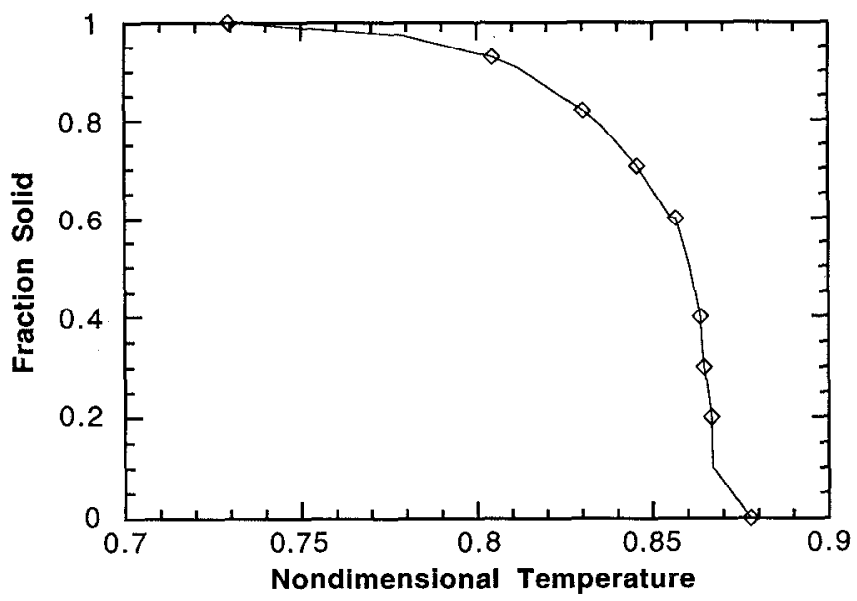

Figure 2. Fraction Solid vs. Nondimensional Temperature at a Rayleigh Number of $4.5 \mathrm{E}+8$

The viscosity values in the fully molten region used were typical for nickel-based superalloys[20]. Between the fully molten region and the fully solid region, the permeability utilized in the computational model must rapidly decrease to values sufficient to completely suppress all fluid motion. Several investigators have measured effective viscosity values of semisolid slurries[21,22,23] and found that effective viscosity values for equiaxed microstructures increase rapidly at solid fractions of 0.4-0.6 for a wide range of shear rates. Bhat et al.[24] and Ganesan et al.[25] have numerically calculated permeability data for cross flow through columnar dendritic microstructures $\left(\mathrm{K}_{\mathrm{r}}\right)$ obtained experimentally for different solid fractions and numerically estimated the flow parallel to the primary dendrite arms $\left(\mathrm{K}_{\mathrm{Z}}\right)$. These researchers provide recommendations of cross-flow and parallel-flow permeability as a function of solid fraction and show that the permeability rapidly decreases at solid fractions of $0.4-0.6$, similar to the viscosity results. In this work, the decrease in convective ability in the mushy zone was simulated by a decreasing permeability, using the values obtained by Ganesan[25] and Bhat[24]. They found that the parallel flow permeability values are consistently higher than the cross flow values which is expected for columnar growth.

The present problem considers the heat transfer that takes place through the ampoule walls of finite thickness. This equation is presented only in its nondimensional form. 
Energy Equation for Crucible Wall:

$$
\operatorname{Pe} \frac{\partial \theta}{\partial \mathrm{z}^{*}}=\frac{\alpha_{\mathrm{c}}}{\alpha_{\mathrm{m}}}\left(\frac{1}{\mathrm{r}^{*}} \frac{\partial}{\partial \mathrm{r}^{*}}\left(\mathrm{r}^{*} \frac{\partial \theta}{\partial \mathrm{r}^{*}}\right)+\frac{\partial^{2} \theta}{\partial \mathrm{z}^{* 2}}\right)
$$

Here, Pe is the Peclet number $\left(v_{\mathrm{g}} \mathrm{L} / \alpha_{\mathrm{m}}\right)$ representing the nondimensionalized growth rate $\left(\mathrm{v}_{\mathrm{g}}\right.$ is the growth velocity), and $\alpha_{c}$ is the thermal diffusivity of the crucible.

A schematic diagram of the computational model is shown in Figure 3. Since the anticipated flows are assumed to be axisymmetric, the modeled region cxtcnds radially from the centerline to the outer edge of the crucible wall. Because it is assumed that the model is quite long in the axial direction, the end effects are neglected. Therefore, the top of the melt is kept at the hot zone temperature and the bottom of the crystal is at the cold zone temperature. The effect of the pull rate is considered by supplying melt at a uniform rate on top and extracting the solidified crystal at a rate equal to the growth rate modified by density differences. At the outer edge of the crucible wall, a temperature boundary condition representative of the radiation and convection characteristics from the crucible wall to the furnace enclosure is imposed. The nondimensional parameters of the growth material, crucible, and furnace conditions are listed in Table 1.

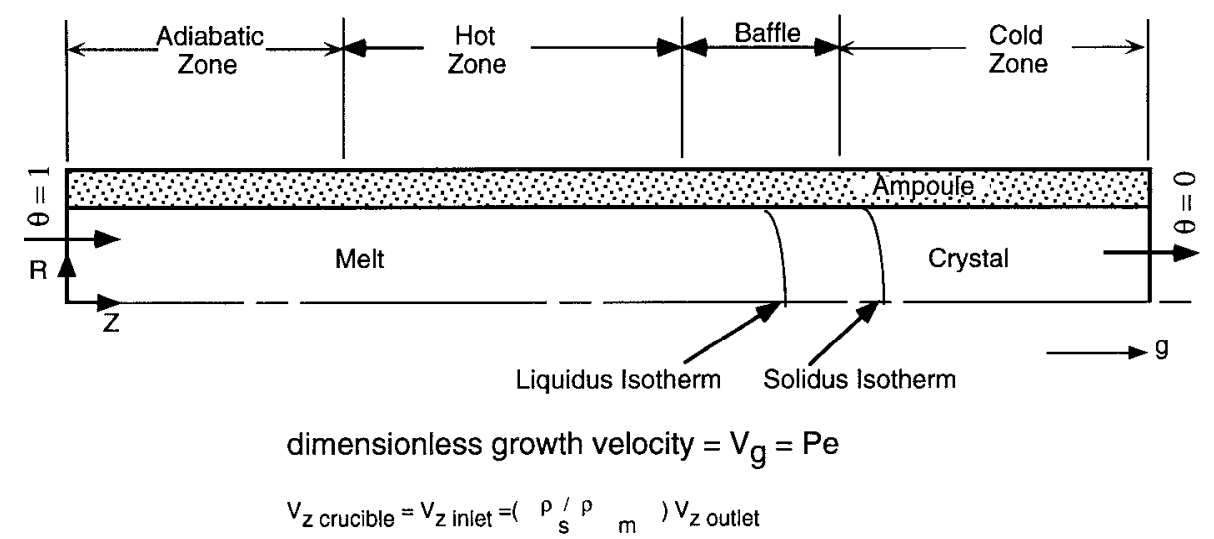

Figure 3. Schematic Diagram of the Extended Length Directional Solidification Model utilized in this study.

\section{Solution Methods}

FIDAP, a finite-element-based, commercial software package, has been used for this simulation. One of the advantages of FIDAP is its ability to handle problems involving heat and mass transfer and latent heat release. Thus, it is especially suited for problems such as this study. The finite element method has the ability to solve complex flow domains and difficult boundary conditions. The first step is to discretize the domain into a set of simply shaped, nine node quadrilateral elements. Along boundaries and surfaces where boundary conditions are to be applied three node quadrilateral elements are used. Within each element the dependent variables are represented as interpolated functions expressed in terms of the values at elemental nodal points. The partial differential equations governing the fluid flow are then replaced by algebraic equations in each element using the Galerkin method, a weighted residual technique. The resulting algebraic equations are solved using the Newton-Raphson method under two convergence criteria conditions to determine the values of the dependent variables throughout the domain. The first criteria is used for the solution vector and the second applies to the 
residual vector. Both criteria in this simulation were set to $0.01 \%$. A solution is obtained when all the variables to be solved meet both convergence criteria.

In the absence of a top adiabatic zone, the model leads to numerical instabilities when either the diameter is increased or the Rayleigh number is increased beyond approximately $10^{6}$. Under these conditions, the upper flow cell increases in both size and intensity. As it approaches the inlet, the strong convection interferes with the ability of the code to enforce the inlet boundary condition.

In order to alleviate these difficulties in the simulation of large diameter crystal growth, the model shown in Figure 3 was used. In Figure 3, an additional adiabatic zone is placed on top of the hot zone. With this geometry, the conditions of the melt inlet are now displaced upwards to the top of the adiabatic zone. This extra length allows the convective streamline cell that forms between the adiabatic zones to grow. Since there is very little change in temperature between the melt inlet and the bottom of the adiabatic zone, the possibility of convective cells forming in this region is quite small. Thus, this extended length model ensures that the upper convective cell will not distort the inlet flow. The extended length model presented here is similar to that used in a previous study [13]. The earlier study, however, considered the solute segregation due to the thermal buoyancy convection and solid-liquid interface deformation of gallium doped germanium (no mushy zone effects were considered). The results of that study compared well with a similar quasi-steady state model of the Bridgman process used by Adornato and Brown[26] which examined the effect of various parameters on solute segregation in the melt. Also, the use of the current set of equations gives excellent agreement with the model results given by Voller and Prakash[27].

The temperature boundary conditions applied to the edge of the crucible werc determined from a separate set of simulation experiments using the commercial finite element code ProCast. Simulations of actual laboratory directional solidification experiments were performed and compared with embedded thermocouple data to determine transient crucible edge temperatures [28]. The thermal boundary conditions thus obtained are shown in Figure 4.

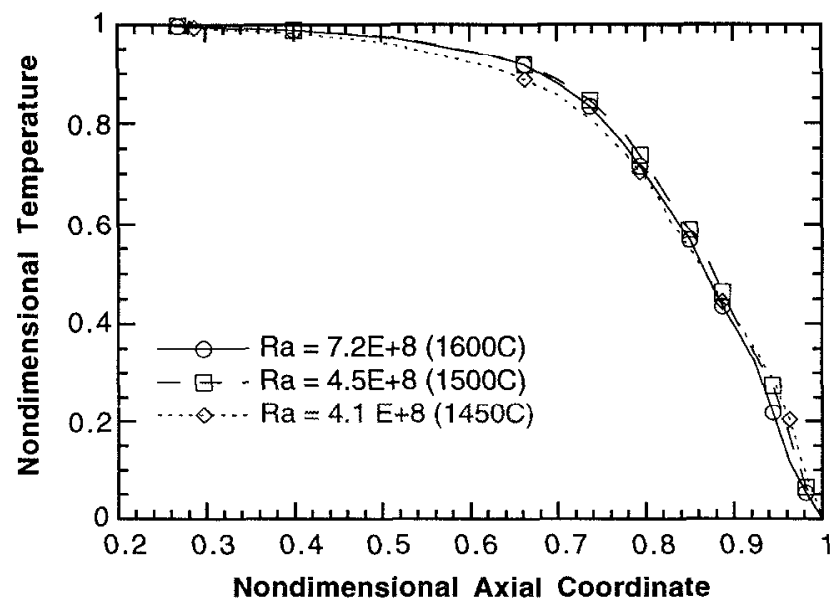

Figure 4. Thermal Boundary Conditions Determined from Thermocouple - Validated ProCast Simulations for the Rayleigh Numbers (Furnace Temperatures as shown)

\section{Results and Discussion}

The primary purpose of this study is to assess the effects of various solidification process parameters (solidification velocity and crucible diameter) in enabling penetration of bulk convection into the mushy zone. The greater the penetration, the greater is the interaction 
of the bulk convection with the lower density interdendritic liquid. Coupling of the thermosolutal buoyancy effects in the mushy zone with the bulk convection effects is neglected due to lack of information on solute partitioning and solutal effects on density. It is the radial component of velocity that causes the circular motion of the fluid into the mushy zone across the dendrites (see Figure 1b). The amount of penetration can thus be quantified by evaluating the nondimensional radial velocity adjacent to the mushy zone. In this paper, the radial velocity at the nondimensional liquidus temperature is the principal figure of merit used to assess convective penetration into the mushy zone.

Typical results for the extended length model are shown in Figure 5 where the effects of solidification velocity are examined for a Rayleigh number of $4.5 \times 10^{8}$. Since axisymmetric flows were assumed, only half of the cells bounded by the centerline and the crucible are shown. The microstructural data by Overfelt[16] and by McLean[29] show that for a constant temperature gradient, increases in the solidification velocity increase the cooling rate and result in a decrease in the primary dendrite arm spacing. This reduction in spacing decreases the tendency for freckles to occur[7]. Figures 5a-5c show the predicted steady state streamlines for Peclet numbers of $0.15,1.50$, and 3.00 (solidification velocities of 0.0005 $\mathrm{cm} / \mathrm{sec}, 0.005 \mathrm{~cm} / \mathrm{sec}$, and $0.01 \mathrm{~cm} / \mathrm{sec}$ ). In addition the nondimensional liquidus and solidus isotherms are shown in each case. Increasing the Peclet number decreases the magnitude of the convection cells, especially the upper cell. Inspection of the predicted position of the mushy zone shows that the equilibrium position moves downward in the pulling direction as the steady-state velocity is increased, as expected. The change in mushy zone position also decreases the penetration of the lower convection cell into the mushy zone. Thus the model predicts that increasing the Peclet number will decrease both the magnitude of the convection cells as well as their penetration into the mushy zone. These results thus indicate a decrease in the potential for freckle forming buoyant plumes as a casting's cooling rate is increased, in agreement with solidification results of Copley et al.[2] and Pollock et al.[7] .

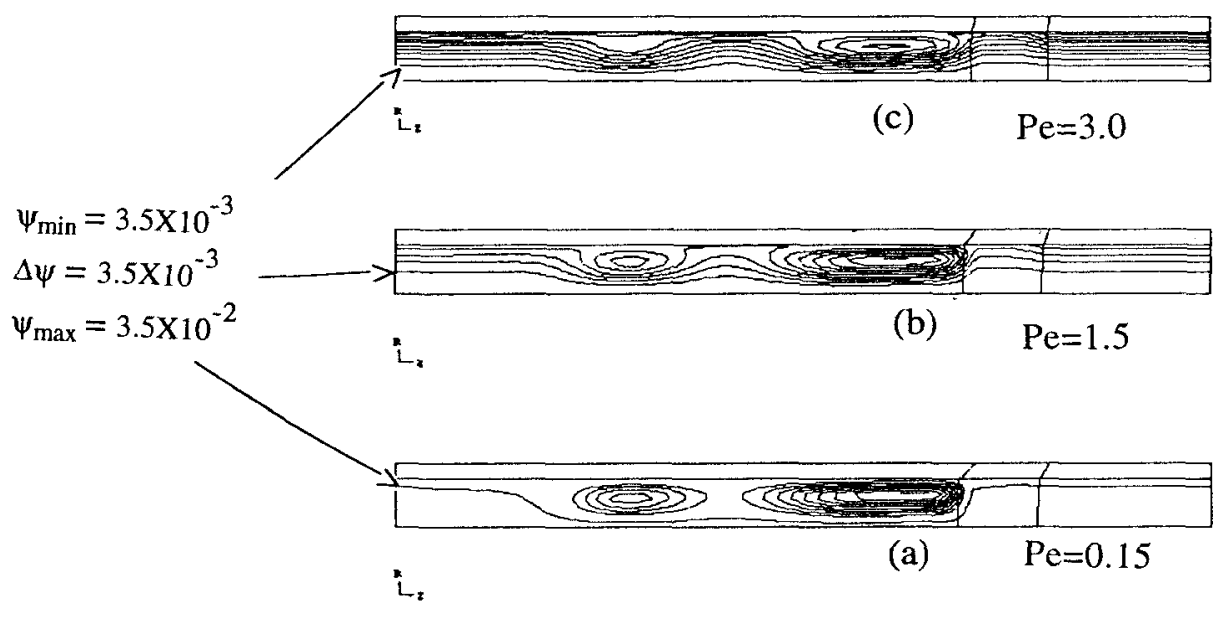

Figure 5. Predicted Steady State Streamlines for a Rayleigh Number of $4.5 \mathrm{E}+8\left(1500^{\circ} \mathrm{C}\right)$ and Peclet Numbers of (a) 0.15 , (b) 1.5 , and (c) 3.0 (Solidification velocities of 0.0005 $\mathrm{cm} / \mathrm{s}, 0.005 \mathrm{~cm} / \mathrm{s}$ and $0.01 \mathrm{~cm} / \mathrm{s}$ ). All arrows point to the minimum streamline value.

Figure 6 shows the nondimensional radial velocity at the liquidus for a Rayleigh number of $4.5 \times 10^{8}$ and a range of Peclet numbers. The values of the nondimensional velocity are most significant in the upper part of the mushy zone which agrees with the results from Heinrich[10] and Felicelli[11]. The nondimensional radial velocity increases from zero at the centerline to a maximum at a nondimensional radial coordinate of 0.03 and drops back to zero at the no-slip crucible wall. Increasing the Peclet number is predicted to decrease the radial component of velocity at the liquidus temperature at all radial positions. The maximum 
nondimensional radial velocity at the liquidus temperature is predicted to decrease from 5 at a Peclet number of 0.15 to 1.9 at a Peclet number of 3.00 . These results are in agreement with the predicted streamlines shown in Figure 5.

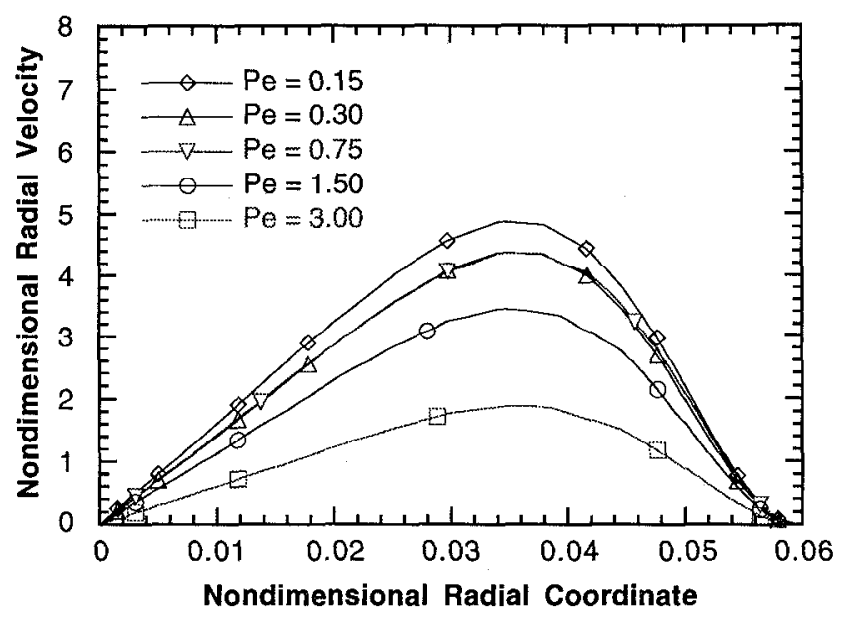

Figure 6. Nondimensional Radial Velocity at the liquidus temperature for a Rayleigh number of $4.5 \times 10^{8}\left(1500^{\circ} \mathrm{C}\right)$ and the Peclet numbers indicated

The effect of crucible diameter at a Peclet number of 0.75 and Rayleigh numbers of $7.2 \times 10^{8}$ and $4.5 \times 10^{8}$ is shown in Figure 7. These two Rayleigh numbers produce average thermal gradients of 90 and $50{ }^{\circ} \mathrm{C} / \mathrm{cm}$ in the samples, respectively. The beneficial effect on the nondimensional radial velocity due to decreasing the Rayleigh number, i.e., hot zone temperature or thermal gradient, is relatively minor. The maximum nondimensional radial velocity only decreases from 4.2 to 3.4 when the Rayleigh number decreases from $7.2 \times 10^{8}$ to

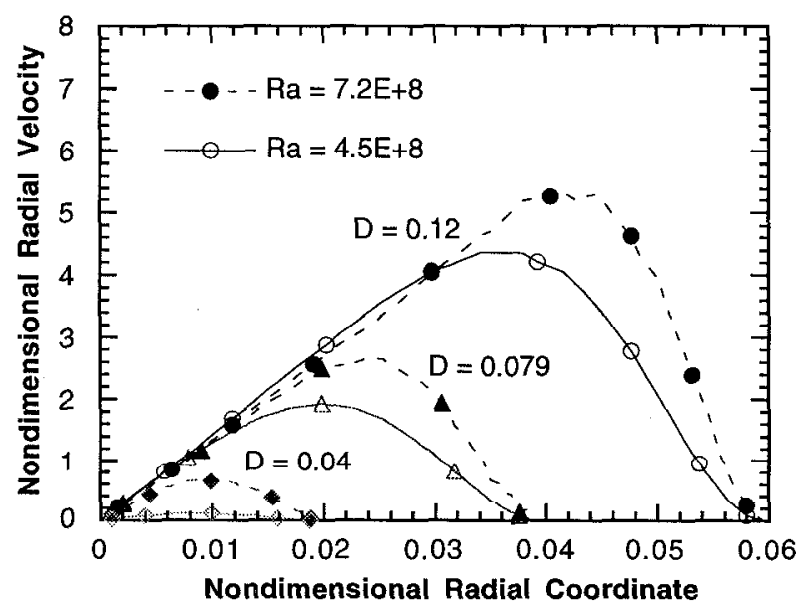

Figure 7. Nondimensional Radial Velocity at the Liquidus Temperature for a Peclet Number of 0.75 (solidification velocity of $0.0025 \mathrm{~cm} / \mathrm{sec}$ ) and the Rayleigh Numbers and Crucible Diameters Indicated. 
$4.5 \times 10^{8}$ (hot zone decreases from $1600^{\circ} \mathrm{C}$ to $1500^{\circ} \mathrm{C}$ ). However, decreasing the crucible diameter from 0.12 to 0.040 decreases the maximum nondimensional radial velocity from 6.1 to 0.1 when the Rayleigh number is $7.2 \times 10^{8}$ (hot zone: $1600^{\circ} \mathrm{C}$ ). A decrease in radial velocity by a factor of 61 is predicted when the crucible diameter decreases by a factor of 3 . Thus the model predicts that decreasing the crucible diameter will decrease the magnitude of the convection cells and their penetration into the mushy zone which indicate a decrease in the potential for freckle forming buoyant plumes. Heinrich [10] also showed the benefits associated with a small diameter casting. His model for a $\mathrm{Pb}-10 \% \mathrm{Sn}$ alloy predicted that for a $1.5 \mathrm{~mm}$ crucible diameter the bulk fluid motion stabilized and all convection was avoided and thus no freckles could occur. This result also agrees with the investment casting foundry experience that thin wall castings are much less susceptible to freckle defects [30].

\section{Conclusions}

An axisymmetric heat and mass transfer model of directional solidification of a typical nickel-based superalloy has been developed. Dimensionless forms of the governing equations were utilized for the computations, which were performed for a number of cases where the Rayleigh number, Peclet number, and crucible diameter have been parametrically varied. The following conclusions can be drawn from the results of the simulations:

(1) Increasing the Peclet number (or solidification velocity) lowers the position of the mushy zone and decreases the magnitude of the bulk convection. This decreases the penetration of bulk convection into the mushy zone which should decrease the potential for freckle forming plumes, consistent with previous experimental results.

(2) Decreasing the crucible diameter significantly decreases the overall convection, including the bulk convection that has penetrated into the mushy zone. This also decreases the potential for freckle formation. This is consistent with previous results and investment foundry experience.

\section{Acknowledgments}

The authors gratefully acknowledge the financial support from NASA's Office of Space Access and Technology under Grant No. NAGW-1192 and Howmet Corporation, Whitehall, MI. In addition, technical discussions with Boyd Mueller of Howmet Corporation, Larry Graham of PCC Airfoils, Inc., and Tony Giamei of United Technologies Research Center were especially helpful.

\section{References}

1. A.F. Giamei, and B.H. Kear, "On the Nature of Freckles in Nickel Base Superalloys," Met. Trans., Vol. 1(1970) 2185-2191.

2. S.M. Copley et al., "The Origin of Freckles in Unidirectionally Solidified Castings", Met. Trans., Vol 1(1970) 2193-2204.

3. A. Hellawell, "The Grain Structure of Castings: Some Aspects of Modeling," in Modeling of Casting. Welding and Advanced Solidification Processes VII, Mark Cross and John Campbell, Eds. (TMS, Warrendale, PA, 1995) 565-576.

4. A.K. Sample, A. Hellawell, "The Mechanisms of Formation and Prevention of Channel Segregation during Alloy Solidification," Mct. Trans., Vol. 15A (1984) 2163-2173.

5. T.D. McCay, and M.H. McCay, "Experimental Measurement of Solutal Layers in Unidirectional Solidification", Journal of Thermophysics, Vol. 2 (July 1988) 197.

6. J.R.Sarazin, and A. Hellawell, "Channel Formation in $\mathrm{Pb}-\mathrm{Sn}, \mathrm{Pb}-\mathrm{Sb}$, and $\mathrm{Pb}-\mathrm{Sn}-\mathrm{Sb}$ Alloy Ingots and Comparison with the System $\mathrm{NH}_{4} \mathrm{Cl}-\mathrm{H}_{2} \mathrm{O}$ ", Met. Trans., Vol. 19A(1988) 1861-71. 7. T.M. Pollock et al., "Grain Defect Formation during Directional Solidification of Nickel Base Single Crystals," in Superalloys 1992, Eds. S.D. Antolovich, R.W. Stusrud, R.A. Mackay, D.L. Anton, T. Khan, R.D. Kissinger, and D.L. Klarstrom, (TMS, Warrendale, Pa 1993) 125-134.

8. Muller, G. , Crystals: Growth, Properties, and Applications, 12, (Berlin, Springer-Verlag) 
9. M.H. McCay, T.D. McCay, and J.A. Hopkins, "The Nature and Influence of Convection on the Directional Dendritic Solidification of a Metal Alloy Analog, $\mathrm{NH}_{4} \mathrm{CL}$ and $\mathrm{H}_{2} \mathrm{O}$ ", Metall. Trans., 24B (August 1993) 669.

10. J.C. Heinrich et al., "Thermosolutal Convection during Dendritic Solidification of Alloys: Part II. Nonlinear Convection,” Met. Trans., 20B(1989) 883-891.

11. S.D. Felicelli, J.C. Heinrich, and D.R. Poirer, "Simulation of Freckles during Vertical Solidification of Binary Alloys", Met. Trans. , 22B(December 1991) 847.

12. S. Motakef, "Interference of Buoyancy-Induced Convection With Segregation During Directional Solidification: Scaling Laws", Journal of Crystal Growth, 102 (1990) 197-213. 13. V. Sahai, J. W. Williamson, and R.A. Overfelt, "Controlling Convection and Segregation by Using Baffles in Bridgman Growth of Large Diameter Crystals at Low Gravity," in Heat Transfer in Microgravity Systems, Edited by S.S. Sadhal and A. Hashemi, ASME, HTDVolume 235(1993) 1-10.

14. R.A. Overfelt et al., "Microstructure and Grain Defect Formation in Superalloys", (proprietary report, Space Power Institute, Auburn Univ. 1995).

15. R. J. Su, W. A. Jemian, and R.A. Overfelt, "Transient Effects In The Directional Solidification Of Al-Cu Alloys", to be published in Journal of Crystal Growth.

16. R.A. Overfelt et al. "Solidification Map of Directionally Solidified Inconel 718", Microstructural Science, Vol. 22.

17. C.A. Wang, Crystal Growth and Segregation in a Vertical Bridgman Configuration, (Ph.D. Thesis, Massachusetls Institute of Technology, 1984).

18. R.E. Taylor, private communication, Thermophysical Properties Research Laboratory, Inc., W. Lafayette, IN, 1995.

19. L. Backerud, G. Chai, and J. Tamminen, Solidification Characteristics of Aluminum Alloys, Vol. 2: Foundry Alloys, (AFS/Skanaluminium,Des Plaines, $\amalg$ 1990)2-15. 20. R.A. Overfelt, C.A. Matlock, and M.E. Wells, "Viscosity of Superalloy 718 by the Oscillating Vessel Technique," submitted to Materials and Metallurgical Transactions: B.

21. D.B. Spencer, R. Mehrabian, and M.C. Flemings, Met. Trans., 3 (1972) 1925-1932.

22. V. Laxmanan, and M.C. Flemings, Metall. Trans., 11A (1980)1927-1937.

23. H. K. Moon, (Ph.D. Thesis, Massachusetts Institute of Technology, 1990).

24. M.S. Bhat, D.R. Poirier, and J.C. Heinrich, "Permeability for Cross Flow through Columnar-Dendritic Alloys," Metall. and Materials Trans., Vol. 26B(1995) 1049-1056. 25. S. Ganesan, C.L. Chan, and D.R. Poirer, "Permeability for Flow Parallel to Dendrite Arms", Materials Science and Engineering, Vol . A151(1992) 97-105.

26. P.M. Adornato, and R.A. Brown, "Convection and Segregation in Directional Solidification of Dilute and Non-Dilute Binary Alloys: Effect of Ampoule and Furnace Design", J. Crystal Growth, 80 (1987) 155-190.

27. V.R. Voller, and C. Prakash, "A Fixed Grid Numerical Modeling Methodology for Convection-Diffusion Mushy Region Phase-Change Problems," International Journal of Heat and Mass Transfer, Vol. 30, (1987) 1709-1719.

28. V. Sahai, P. Banerjee, and R.A. Overfelt, unpublished research 1995.

29. M. McLean, Directionally Solidified Materials for High Temperature Scrvicc, (London, The Metals Society, 1983).

30. B. Mueller, private communication, HOWMET Corporation, Whitehall, MI, 1995. 